International Journal of Engineering \& Technology, $7(2.31)(2018) 240-244$
International Journal of Engineering \& Technology
WPC
Website: www.sciencepubco.com/index.php/IJET
Research paper

\title{
Medimate : Ailment Diffusion Control System with Real Time Large Scale Data Processing
}

\author{
S. Sujeetha ${ }^{1^{*}}$, Veneesa $\mathrm{Ja}^{2}, \mathrm{~K}$. Vinitha ${ }^{3}$, R. Suvedha ${ }^{4}$ \\ ${ }^{1}$ Assistant Professor, Sri Sairam Institute of Technology, Chennai. \\ ${ }^{2}$ Sri Sairam Institute of Technology, Chennai. \\ E-mail:veneesajayavel@gmail.com \\ ${ }^{3}$ Sri Sairam Institute of Technology, Chennai. \\ E-mail:kanyadhariveena@gmail.com \\ ${ }^{4}$ Sri Sairam Institute of Technology, Chennai. \\ E-mail:r.suvedha11@gmail.com \\ *Corresponding author E-mail:sujeetha.it@sairamit.edu.in
}

\begin{abstract}
In the existing scenario, a patient has to go to the hospital to take necessary tests, consult a doctor and buy prescribed medicines or use specified healthcare applications. Hence time is wasted at hospitals and in medical shops. In the case of healthcare applications, face to face interaction with the doctor is not available. The downside of the existing scenario can be improved by the Medimate: Ailment diffusion control system with real time large scale data processing. The purpose of medimate is to establish a Tele Conference Medical System that can be used in remote areas. The medimate is configured for better diagnosis and medical treatment for the rural people. The system is installed with Heart Beat Sensor, Temperature Sensor, Ultrasonic Sensor and Load Cell to monitor the patient's health parameters. The voice instructions are updated for easier access. The application for enabling video and voice communication with the doctor through Camera and Headphone is installed at both the ends. The doctor examines the patient and prescribes themedicines. The medical dispenser delivers medicine to the patient as per the prescription. The QR code will be generated for each prescription by medimate and that QR code can be used forthe repeated medical conditions in the future. Medical details are updated in the server periodically.
\end{abstract}

Keywords: Heart beat sensor, temperature sensor, load cell, RTP, ultrasonic sensor, QR code, dispenser, medimate.

\section{Introduction}

People in rural areas generally have less medical facilities than their urban counterparts. On average, these individuals have to travel five times the distance to access these medical services. By the advanced tools, we can promote faster analysis, decreased pain and less expenditure, which results in saving more lives. Our system will ensure to prevent spread of diseases through the application of Big Data Technologies and IoT Sensing. It is a groundbreaking approach to implement technology in the healthcare industry. Our goal line is to distribure vaccines or preventive medicines during an out break of endemic diseases and to ensure $24 / 7$ availability of doctors even in rural areas. Medimate is more efficient in fetching accurate parameters of the patient with the help of sensors [2] like Heart Beat Sensor, Temperature Sensor and Ultrasonic Sensor for measuring height and Load Cell for weight measurement. Hence doctors can easily diagnose the medical complaints of the patient and make the prescriptions accordingly. Prescribed medicines are dispatched through dispenser [1]. QR code will be generated which can be used to dispense the medicines for repeated conditions.

\section{Literature Survey}

[1] The dispenser is used for dispatching the prescribed medicines. Dispenser contains slots where each slot is filled with the specific medicine. The dispenser is checked periodically in-order to refill the emptied tablets and remove the expired tablets. Medicine dispenser will dispatch the medicines prescribed by the doctor with the correct dosage to the patient [1]. Then the patient will collect the tablet from the dispenser outlet tray.

Medimate is configured with basic health monitoring parameters for diagnostic purpose. [2] Heartbeat sensor is used for measuring the pulse rate of the patient, load cell is used for measuring the weight of an individual, the body temperature of the patient is noted by the temperature sensor and ultrasonic sensor is used for the height of an individual. These are the basic health monitoring parameters configured in the medimate for diagnosing the patient health condition [2].

Medimate is configured with a camera and headphones for the purpose of communication [3]. The patient consults with the doctor via video conferencing through headphones and camera. The application for enabling video conference is installed at both the ends. [3] This promotes a face to face interaction between the doctor and the patient. 
A database is used to store the records and transactions of each and every process of the medimate into the main server [4]. This will reduce the expenditure spent on the physical storage [4]. The patient health history and the medicinal data are maintained in the database. The patient history can be retrieved for future use.

The QR code is obtained with a wide amount of information. The QR code will be generated for each prescription by medimate [5]. The QR code generated by the medimate can be used for the repeated medical conditions in the future. This can be initiated by setting-up the QR code reader in the medimate. Medical details are updated in the server periodically.

\section{System Model}

The patient visits the medimate and initialize its activity. The activity is guided by the voice over communication in which the system is recorded with rural language of the state and a common foreign language that helps the patient to understand the procedure to operate the machine. The patient's health parameters are measured by the respective sensors [2].

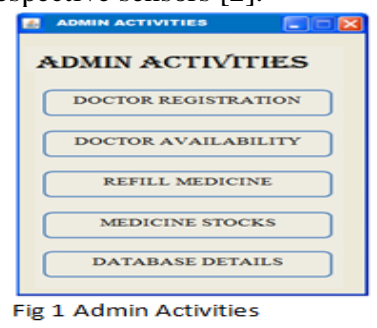

Incase of heart beat sensor the pulse rate of the patient is analyzed and similarly the load cell calculates the patient's weight, temperature sensor measures the body temperature of the patient, ultrasonic sensor is for identifying the height of an individual and these parameters are stored in the database [4] which is sent to the doctor and it is also used for retrival of the patient's history. After all these procedures the patient is guided to initialize the face to face communication [3] through video conferencing with the doctor who is available online then, with the help of camera and headphones.

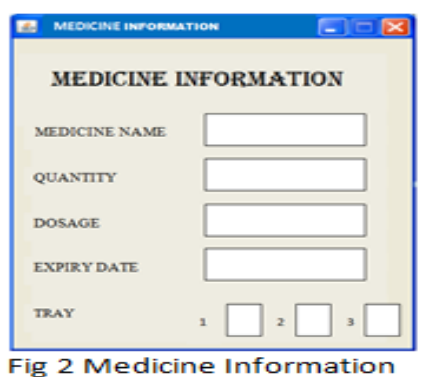

Patient is provided with the list of options to choose the convenient language for the purpose of communication so that the doctors who are in online and those who fall within the given category of that language are listed on the system along with their field of specialization.

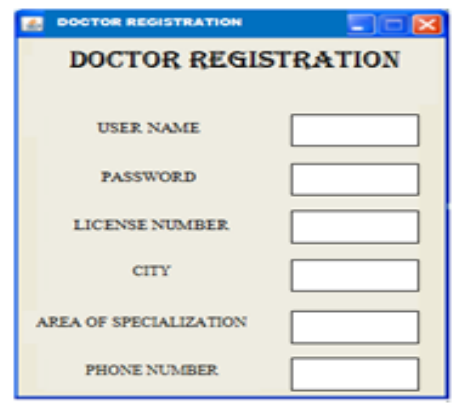

Fig 3.1 Doctor Registration
This option is enabled because if the patient already knows what they are undergoing, they could select that specific doctor who could help them with their problem and prescribes medicine. Once the communication is set, the data that is collected about the patient is sent to the doctor who is on the other end of the communication line. The problem will be diagnosed by the doctor and the doctor checks for the availability of medicines in the patient's local machine and prescribes that medicine by selecting them. The medicine is dispatched to the patient through the dispenser [1] along with the QR code which is generated by the medimate.

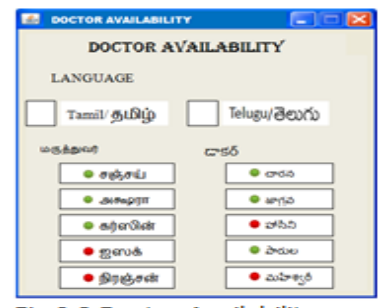

Fig 3.2 Doctor Availability

The patient collects the medicine and QR code from the medimate. The QR code [5] can be used for repeated medical conditions in the future. Thus the communication is terminated after a complete process of consultation with the doctor.

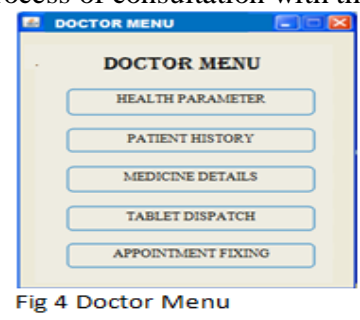

The Server will monitor both the users' information in its database and verify if required. The admin register with new doctors and takes care about the availability of medicine stocks and their expiry date. Figure 1 clearly explains the activities done by an admin. The doctors then register with the system and the registration details include the design fields like Username, License number, city, Password, Phone number and other information like their field of specialization. They can access the system with their user name and password. Figure 2 shows the menu in which the medicine informationsare entered into the database. The doctor registration page will be as that of Figure 3.1 .

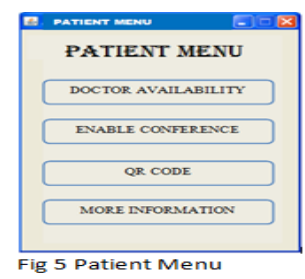

Figure 3.2 is used to display the availability of doctors to the patient. Patient is allowed to select their comfortable language. Red and green color specifies the availability of the doctor.Doctor menu and patient menu is exhibited through Figure 4 and Figure 5 respectively. Patient menu will have details like their health parameters, dispatching medicine and scanning QR code. Doctor menu will have informations about patient's health parameters, medicine details and the have botton for dispatching the medicine. 


\section{System Architecture}

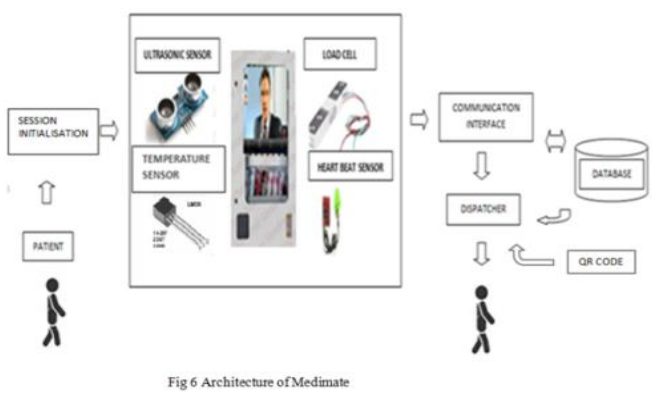

\section{Session Initialisation}

The complete system will be initialised during their set up. After that it will enter into the power saving mode to reduce the power consumptions. Every time the patient visits the medimate the session will be initialised. The system will be activated from the power saving mode when the patient intimates their presence by scanning the RFID provided to them. The patient has to receive their RFID initially from the hospital.

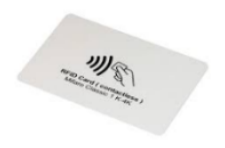

\section{Heart Beat Sensor}

Heartbeat sensors are based on the photo phlethysmography and they are used for providing digital outputs of pulse rate when a finger is placed. Figure 6.1 explains the structure of heartbeat sensor. A sensor and a control circuit are the basic components of a simple heartbeat sensor. The sensor consists of an IR LED and a Photo Diode placed in a clip where as the Control Circuit consists of an Op-Amp IC and a few other modules that help in connecting the signal to a Microcontroller. The source of light would be an IR LED and the detector would be any Photo Diode or a Photo Transistor. Every heartbeat will alter the amount of blood in the finger. A very high resistance resistor R1 is being used, because most of the light through the finger is absorbed. With every heart pulse, detector signal differs. The difference is transformed into electrical pulses. These signals are amplified as well as generated via amplifiers that output $+5 \mathrm{~V}$ logic level signals. Output signals are also characterized by an LED that flashes at every heartbeat.

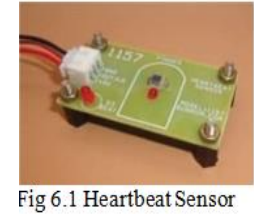

\section{Temperature Sensor}

The LM35 is a combined circuit sensor that can be used to identify temperature with an electrical output relational to the temperature. Figure 6.2 gives the structure of temperature sensor. It can measure temperature more precise than using a thermistor. The LM35 produces a greater output voltage than thermocouples and may not need that the output voltage be amplified. The LM35 has an output voltage that is relational to the Celsius temperature. $-55^{\circ} \mathrm{C}$ to $150^{\circ} \mathrm{C}$ is the operating temperature range. In response to every ${ }^{\circ} \mathrm{C}$ rise/fall in ambient temperature the output voltage varies by $10 \mathrm{mV}$. The scale factor is $0.01 \mathrm{~V} /{ }^{\circ} \mathrm{C}$. The $\mathrm{LM} 35$ does not need any exterior calibration or pruning and preserves an accuracy of $+/-0.4^{\circ} \mathrm{C}$ at room temperature and $+/-0.8^{\circ} \mathrm{C}$ over a range of $0^{\circ} \mathrm{C}$ to $+100^{\circ} \mathrm{C}$.

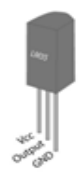

Fig 6.2 Temperature Sensor

\section{Load Cell}

For weight measurement, we use load cell. The structure of the load cell is explained in Figure 6.3. The load cell has low-profile, developed accuracy and may be used for off center loading. The load cell is also constructed for high capacity OEM applications as well as provided in a huge range of competencies from gram ranges to $500 \mathrm{Ibs}$ in the same form fit function. It has been a perfect option for several medical applications. Through the usage of load cells, we may assess the calibrated load of kilograms is in current form, so I to $\mathrm{V}$ converter is utilized for transforming it into a voltage form. Then the precision rectifier is utilized.

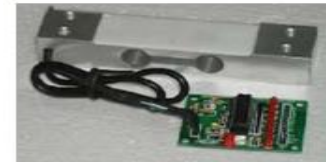

Fig 6.3 Load Ce11

\section{Ultrasonic Sensor}

High frequency sound waves are generated by ultrasonic sensors and it evaluates the echo which is received back by the sensor. Figure 6.4 explains the structure of ultrasonic sensor. The time interval is calculated by the sensors by sending the signal and receiving the echo to determine the height of an object. The transducer is used by the system to produce sound waves in the ultrasonic range, above 18,000 hertz. It converts electrical energy into sound waves, then it converts the sound waves into electrical energy upon receiving the echo which can be measured and displayed.

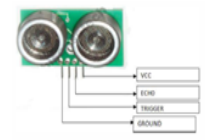

Fig 6.4 Ultrasonic Sensor

\section{Communication Interface}

The application is installed at both the ends for the purpose of voice communication and chatting with doctor regarding health complaints. Voice and video based communication between doctor and patient is being implemented using RTP protocol.

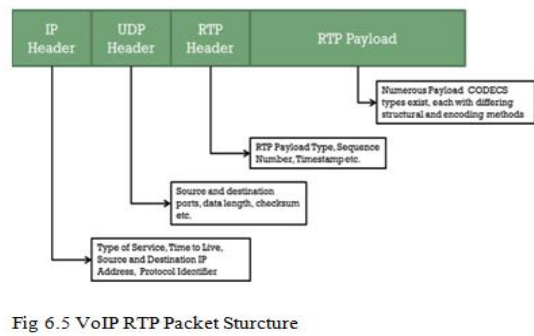

RTP is a transport protocol for real time applications,its structure is in Figure 6.6. RTSP/RTP will communicate via 1 port for command and data. That is the RTSP port. UDP enables large packet transfer. VoIP faces backoffs in TCP hence UDP is reliable. 


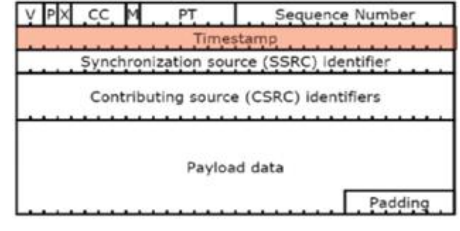

Fig 6.6 RTP packet format

\section{Dispenser}

The dispenser is a metal device which has several partitions for a variety of medicines. The dispenser will get the instructions in the form of digital signals. After receiving the signals the motor will rotate and the tray will move in order to dispatch the medicines. The input to the dispenser will be given in two different manners: 1. Directly from the user (doctor) 2. Through the QR code reader. Through QR code prescription will be retrieved, hence the dispenser will dispatch the respective medicines.

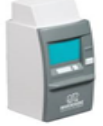

Fig 6.7 Dispenser

\section{QR Code}

QR Code will be generated with patient's ID given in their respective RFID. The details about the patient and the medicine will be encrypted and then QR code will get generated which encloses these details. The validity will be set during the generation of $\mathrm{QR}$ code. The $\mathrm{QR}$ code when scanned will be decrypted and the details will be retrived. When this QR code is scanned patient can dispense the medicines without consultation with doctor

Patient history and informations will be stored in the database The database contents like patient information will be encrypted using RSA and will be decrypted and retrieved using AES algorithm.

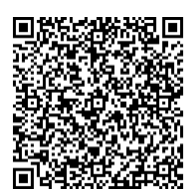

\section{Experimental Works}

The experimental results show that medimate is much better than the existing, since waiting time of the patient has been reduced. The access is made simple and easier for the rural people through recorded voice instructions. User details are collected, monitored and maintained by the server in their database. The connection between the user and the doctor is established by the server. The Server will update each patient's information after each activity in the database. Medicine details are also updated in the server. The medicines are dispatched by the dispenser based on the prescriptions. QR code will also be generated by the system that can be used for repeated medical conditions in the future.

\section{Comparison of System}

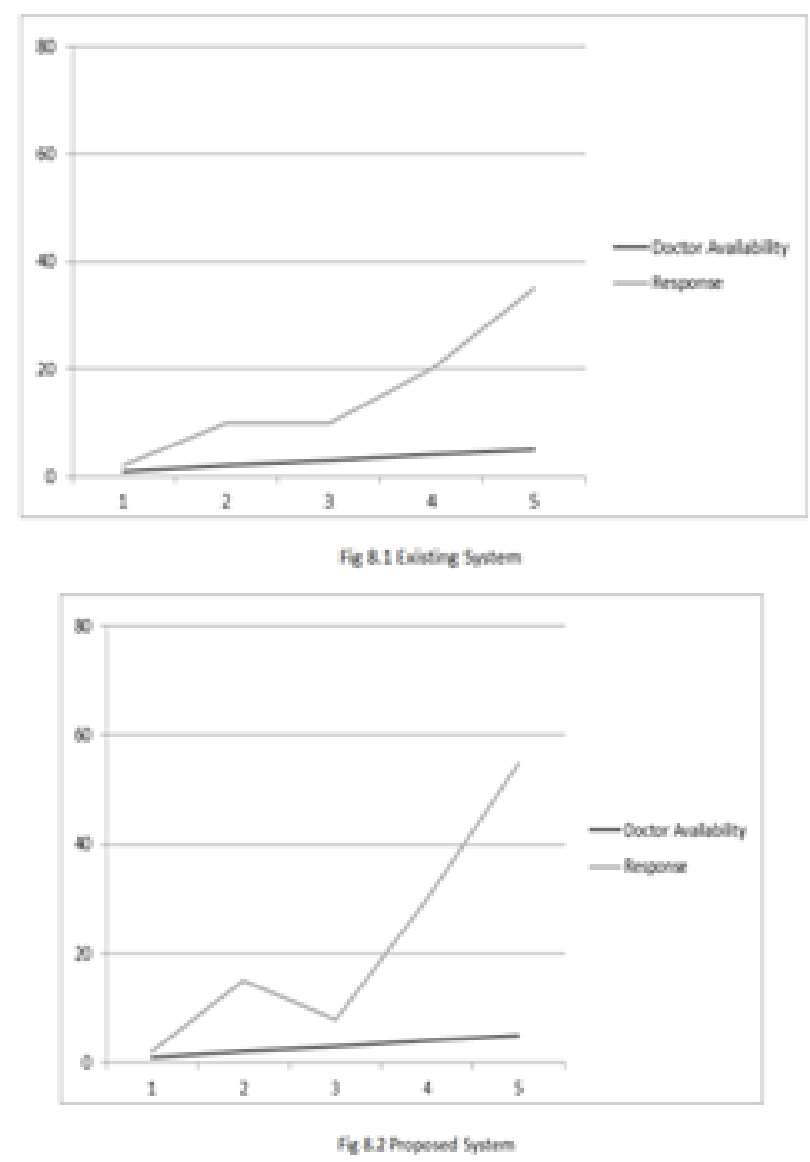

Figure 8.1 shows the existing system scenario, where the rural people will not have sufficient healthcare facilities and doctors. From Figure 8.2, we can conclude that our proposed system is efficient than the existing system since $24 / 7$ availability of the doctor is enhanced

\section{Future Works}

Future work will be employing fingerprint sensors to promote the security of medimate. The patient history can be easily retrieved by using the fingerprint sensor. Fingerprints will be unique for each individual, hence consistency will be high. Alerts can be sent to the nearby ambulance service, the location of the patient can be tracked using the GPS (Global Positioning System) in case of emergency. Blood Glucose Sensor can be used to measure the sugar level of an individual. Instead of manual inputs, voice inputs can be included to make the process easy and simple for the rural patients. The remainders for medicine intake can also be sent to the patients registered mobile phone, this will be helpful for an elderly patient. Even the remainders for checkups can also be sent.

\section{Conclusion}

Thus the implementation of Medical ATM (ATM based health care monitoring system) for the benefit of rural people is done. Various health parameters like pulse rate, temperature, height and weight are measured using heartbeat sensor, temperature sensor, ultrasonic sensor and load cell respectively. The video conference is being enabled using the RTP (Real-time Transport Protocol). The medicines are dispatched by the dispenser as per the prescription. This system eliminates the time wastage in the hospitals and in the medical shops. It supports the concept of Digital India since it excludes the use of paper and pen for maintaining the patient records. 


\section{References}

[1] Ilapakurti A, Vuppalapati JS, Kedari S, Kedari S, Chauhan C \& Vuppalapati C, "iDispenser-Big Data Enabled Intelligent Dispenser", IEEE Third International Conference on Big Data Computing Service and Applications (BigDataService), (2017), pp. 124-130

[2] Sarkar S \& Saha R, “A futuristic IOT based approach for providing healthcare support through E-diagnostic system in India”, Second International Conference on Electrical, Computer and Communication Technologies (ICECCT), (2017), pp.1-7.

[3] Priyadharshini J, "Development of embedded web server for health care system using E-card", International Conference on Algorithms, Methodology, Models and Applications in Emerging Technologies (ICAMMAET), (2017), pp.1-5.

[4] Sangavi RS, Reddy TS, Prabhu TM \& Kumar AJ, "AMM-an Automated Medical Machine enabling enhanced features for telemedicine using cloud computing", International Conference on Information Communication and Embedded Systems (ICICES), (2013), pp.125-129.

[5] Uzun V, "R-code based Hospital Systems for Healthcare in Turkey", IEEE 40th Annual Computer Software and Applications Conference (COMPSAC), (2016), pp.71-76. 